\title{
Dynamic Radio Resource Allocation for Macro-Femto Hybrid Cellular Network Maintaining Fairness
}

\author{
Md. Samee Ullah Chowdhury ${ }^{1}$, Shamit Chakraborty ${ }^{1}$, Tanveer Arif Nipu ${ }^{1} \&$ M. Shamim Kaiser ${ }^{2}$ \\ ${ }^{1}$ Department of Electrical and Electronic Engineering, BRAC University, Dhaka, Bangladesh \\ ${ }^{2}$ Institute of Information Technology, Jahangirnagar University, Dhaka, Bangladesh \\ Correspondence: Md. Samee Ullah Chowdhury, Department of Electrical and Electronic Engineering, BRAC \\ University, Dhaka, Bangladesh. E-mail: samee.chy@gmail.com
}

Received: December 30, 2011 Accepted: January 11, 2012 Online Published: June 1, 2012

doi:10.5539/nct.v1n1p67

URL: http://dx.doi.org/10.5539/nct.v1n1p67

\begin{abstract}
Macro-Femto Hybrid Cellular Network (MFHCN) has been considered as one of the most promising infrastructure for the upcoming next generation cellular network. Femto-Cell (FC) is very effective when it comes to reducing poor signal coverage. The range of the device might be low but it functions to reduce the traffic congestion of the larger Macro Network as well as provide enhanced data rate for both voice and data transmission. In short, it provides better Quality of Service (QoS), i.e., reduce congestion, increase capacity, reduce system outage, while using minimal power consumption. In this work we propose a suboptimal Resource Allocation algorithm for MFHCN. Multiuser and Multiservice are considered in this proposed model. A priority parameter is introduced to maintain fairness amongst all users. By doing so, we were successful in achieving improved outage probability without compromising the total system throughput.
\end{abstract}

Keywords: Femto-Cell, radio Resource Allocation, MFHCN, outage probability, fairness

\section{Introduction}

A typical human behavior is that human beings prefer reliability. Every individual's daily schedule now demands the availability of technologies whenever needed. If we look at the usage of the internet in our daily lives, even the prominent concept of Third Generation (3G) internet, which has been so successful in fulfilling everyone's need, is eventually not good enough if it is not accessible to the user whenever he/she demands, either due to being on the cell edge area or indoors (e.g. basement). For this reason many researches are being conducted to enhance the system capacity while using the current technology. During our literature survey we came across a few other probable solutions. One of them is by increasing the total transmission power of the system (Ahamed, 2008). But since we are using Microwaves for transmission, so if we exceed a certain limit, it will cause human health hazard (Revermann, 2002) and also cause wastage of power. Another solution that we came across is the use of Cooperative Relay System (Kaiser, 2010). But the usage of such a technology introduces additional propagation delay into the system, which cannot be tolerated in case of Real Time (RT) users. After different reviews and logics, we are considering to use of FCs as an efficient solution in terms of fairness and increasing the total system capacity.

FC is a very small radius, low power base station which is very user friendly due to its plug-and-play feature and can use the same transmission frequency as of that of the base station (Holger, 2008). But due to this it introduces Co-Channel Interference (CCI) into the system which can be mitigated by using several techniques, one of them is Frequency Reuse (FR), another one is the Inter-Cell Coordination (ICC) scheme and the other one is Fractional Frequency Reuse (FFR) technique (Boudec, 2009; Stolyar, 2008; Elayoubi, 2008; Assaad, 2008; Necker, 2007; Simonsson, 2007). Hence our work is more focused on Resource Allocation.

One of the primary objectives of our work is to build a MFHCN that will ensure "Fairness". The idea of Fairness has multiple definitions (Ahmed, 2009), one of them is, every Mobile Users (MU) will receive equal data rate despite their channel conditions. Another term is called "Maximum-Minimum Fairness" (Wikipedia, 2011), in which it is mentioned that, regardless of a minimum data rate, best effort is made to make sure that the data rate is maximized as much as possible. Amongst all, we preferred to define fairness in such a way where a MU having ideal channel condition will receive preference and vice versa, thus increasing total system capacity. In addition to that, the power allocation done within the entire MU will be such that every mobile user will receive 
their minimum desired Data Rate (DR) regardless of their channel conditions and we plan to achieve this by using our own Priority Equation about which will be discussed later on. If Resource Allocation is carried out while maintaining a low level of fairness then despite having a high average system throughput the system will be facing low stability when it comes to QoS. Furthermore, without the fairness factor, these cell edge users will be getting deprived as they will be exposed to high signal attenuation, which means they will require more power in order to maintain their desired DR (Boudec, 2009). Here the priority equation will play its part by making sure that the cell edge users will be given more power, keeping in mind that the additional power provided does not deplete too much of the total power available for the network.

We will be using the Orthogonal Frequency Division Multiple Access (OFDMA) technique since it is considered to have enhanced capability in comparison with Code Division Multiple Access (CDMA) and Time Division Multiple Access (TDMA) (Yin, 2006), and as only the downlink scenario in MFHCN is considered in our system, this technique has been preferred to be adapted in the next generation wireless network since it is anticipated to enhance both the coverage and capacity of the network (IEEE 802.16m-07/002r4, 2011; Yeh, 2008). It has also been mentioned that the use of OFDMA provides a high transmission rate, enhances the frequency efficiency and cancels out multi-path fading (Zhou \& Sato, 2009).

For power allocation we will be using Multi-level Water Filling Algorithm (MLWFA) which is a modified version of Water-Filling Algorithm (WFA). In WFA, a sub-carrier with a low noise variance or higher SNIR is allocated more power (Yu, 2001; Flanagan, 2003), but the primary reason for choosing MLWFA is because the power is allocated up to a certain limit for every user so that a particular user does not interfere with its neighboring ones (Zou, 2010). The computational complexity of optimal power allocation is extremely high. Thus suboptimal algorithm has been proposed (Kaiser, 2010). The main focus of our work is to apply one such power allocation scheme which will maintain fairness amongst the users and devise an optimization problem subjected to total power and QoS. The proposed priority parameter will be maintaining the fairness while allocating resources (power and sub-carrier) to the MU. Our goal is to improve the Outage Probability without compromising the Total System Throughput. The parameters consist of the full channel state information and the QoS constraint.

Section 2 discusses the system model, section 3 includes the optimization problem, section 4 shows the simulations and results, and finally we conclude our work in section 5 along with some future recommendations

\section{System Model}

In this proposed system model, one Macro-Cell (MC) is considered, and the FCs are placed at the cell-edge area of that MC coverage area since we are considering the worst case scenario for our work. We are also considering this system to be an OFDMA downlink system. The MC Base Station (M) is connected with a total $i$ number of both RT and NRT users. It is assumed that all the Mobile Base Stations (MBS) know the delay (maximum tolerable time delay, $\tau_{\max }$ ) and channel state information (minimum data rate, $R_{\min }$ and maximum tolerable interference, $\left.I_{\max }\right)$. Our cell is a single antenna cell and the only form of fading we are considering is the Rayleigh Fading.

\subsection{SNIR of Macro-Cell}

The MBS hands off to the FC if and only if the SNIR of Femto-Cell $\left(S N I R_{F}\right)$ is greater than the SNIR of Macro-Cell $(S N I R M)$. The SNIR of the $k$-th user using $n$-th sub-carrier of MC are calculated in the following way.

$$
\operatorname{SNIR}_{M}=\frac{P_{k, n} G D^{-\alpha_{m}}}{\sigma^{2}+\sum p_{k, n}^{*} g^{*} d^{*-\alpha_{M F}}}
$$

Here $P_{k, n}$ is the power allocated to the $k$-th user using $n$-th sub-carrier of the MC, $G$ is the channel gain, $D$ is the distance between the BS to the user where $\alpha_{m}$ is the loss exponent which vary from 2 to $3.5, \sigma^{2}$ is the noise variance, $p_{k, n}^{*}$ is the interfering FC power, $g^{*}$ is the channel gain of the interfering FC, $d^{*}$ is the distance between the MBS and the interfering FC, and $\alpha_{M F}$ is the loss exponent of Macro user to the interfering FC which also varies from 2 to 3.5 .

So we can rewrite Equation (1) as,

where, $I=\sum p_{k, n}^{*} g^{*} d^{*-\alpha_{M F}}$.

$$
S N I R_{M}=\frac{P_{k, n} G D^{-\alpha_{m}}}{\sigma^{2}+I}
$$




\subsection{SNIR of Femto-Cell}

The SNIR of the $k$-th user using $n$-th sub-carrier of FC can be calculated in the following way.

$$
\operatorname{SNIR}_{F}=\frac{p_{k, n} g d^{-\alpha_{F}}}{\sigma^{2}+\sum p_{k, n}^{*} g^{*} d^{*-\alpha_{F F}}+P_{k, n} G D^{-\alpha_{M F}}}
$$

Here, $p_{k, n}$ is the active FC transmission power, $g$ is the channel gain of the FC, $d$ is the distance between the FC BS to the user, $\alpha_{F}$ is the loss exponent of that $\mathrm{FC}$ user which varies from 2 to $3.5, \sigma^{2}$ is the noise variance, is $p_{k, n}^{*}$ the interfering FC power, $g^{*}$ is the channel gain of the interfering FC, $d^{*}$ is the distance between the FC user to the interfering FC, $\alpha_{F F}$ is the loss exponent of Macro user to the interfering FC which also varies from 2 to 3.5, $P_{k, n}$ is the interfering MC power, $D$ is the distance between the FC user to the MC BS and $\alpha_{M F}$ is the loss exponent of MC.

So, we can rewrite the Equation (3) as,

$$
\operatorname{SNIR}_{F}=\frac{p_{k, n} g d^{-\alpha_{F}}}{\sigma^{2}+I_{F}}
$$

where, $I_{F}=\sum p_{k, n}^{*} g^{*} d^{*-\alpha_{F F}}+P_{k, n} G D^{-\alpha_{M F}}$

\subsection{Data Rate of $M B S$}

Now, the data rate of MC user can be expressed as,

$$
B_{k, n}=\log _{2}\left(1+\frac{P_{k, n} G D^{-\alpha_{m}}}{\sigma^{2}+I}\right)
$$

and the data rate of FC user can be expressed as $b_{k, n}$,

$$
b_{k, n}=\log _{2}\left(1+\frac{p_{k, n} g d^{-\alpha_{m}}}{\sigma^{2}+I_{F}}\right)
$$

Here, $P_{k, n}$ and $p_{k, n}$ are the transmit power of the MC and FC BS respectively. This cannot exceed the total power of $P_{k, n}+p_{k, n} \leq P_{k}$. ( $P_{k}$ is the total power we are considering.)

\section{Optimization Problem}

Our concern is to improve the outage probability and maximize the data rate of the system by allocating the resources (Power and Subcarrier) in such a way that the QoS and Fairness will be maintained. So the optimization problem for the MC user can be written as:

$$
\begin{aligned}
& \text { Maximize } \sum_{k} \sum_{n} B_{k, n} \\
& \text { Subject to } \sum_{k} \sum_{n} P_{k, n} \leq \sum P_{k} \\
& \qquad P_{k, n}>0 ; \quad P_{k, n} f^{2} \leq I_{\max }
\end{aligned}
$$

Here $f$ is the channel gain of the MC user. So the term $P_{k, n} f^{2} \leq I_{\max }$, makes sure that the current user does not interfere with its neighboring users as according to the MLWFA.

For the FC user, the optimization problem can be written as,

$$
\begin{gathered}
\text { Maximize } \sum_{k} \sum_{n} b_{k, n} \\
\text { Subject to } \sum_{k} \sum_{n} p_{k, n} \leq \sum P_{k} \\
p_{k, n} g^{2} \leq I_{\max }
\end{gathered}
$$


Here $g$ is the channel gain of the FC user and $I_{\max }$ is the maximum tolerable interference. So the term $p_{k, n} g^{2} \leq I_{\max }$ makes sure that the current user does not interfere with its neighboring users as according to the MLWFA.

So,

$$
\text { total system data rate }=\sum B_{k, n}+\sum b_{k, n}
$$

\subsection{Priority Equation}

As we have stated above, our main aim is to improve the outage probability and increase the total system DR. So, to achieve our goal we introduce a priority parameter. It is given as,

$$
\Psi=\left[\left[\left(\frac{R_{\min }}{R(t)}\right)^{\gamma} *\left\{\frac{\tau(t)}{\tau(t-1) * \tau_{\max }}\right\}^{\beta}\right]+\beta\right]^{\Delta}
$$

where, $R_{\min }$ is the minimum data rate of the user, $R(t)$ is the current time slot data rate, $\tau(t)$ is the current time slot delay, $\tau(t-1)$ is the previous time slot delay, $\tau_{\max }$ is the maximum tolerable delay, $\gamma, \Delta$ and $\beta$ is a constant which varies between 0 and 1 depending on the situation.

As the delay is a big factor especially in case of RT MUs', at first it is checked whether $\tau(t) \geq \tau_{\max }$ or not. If yes, then $\beta$ is set as 1 and $\gamma$ set as 0 . Then we calculate $\Psi$ as per Equation (10). Now, if it is greater than one, it will get high priority. If the situation is the other way around, then $\beta$ set as 0 . Then we will check whether $R(t)<R_{\min }$ or not. If yes, then $\gamma$ is set as 1 and then the priority value is calculated. If vice versa, then $\gamma$ set as 0 . After four loops, if it still fails to meet the desired SNIR then we will set $\Delta$ as 0 which means it will not get priority. The entire process is better demonstrated by Figure 1.

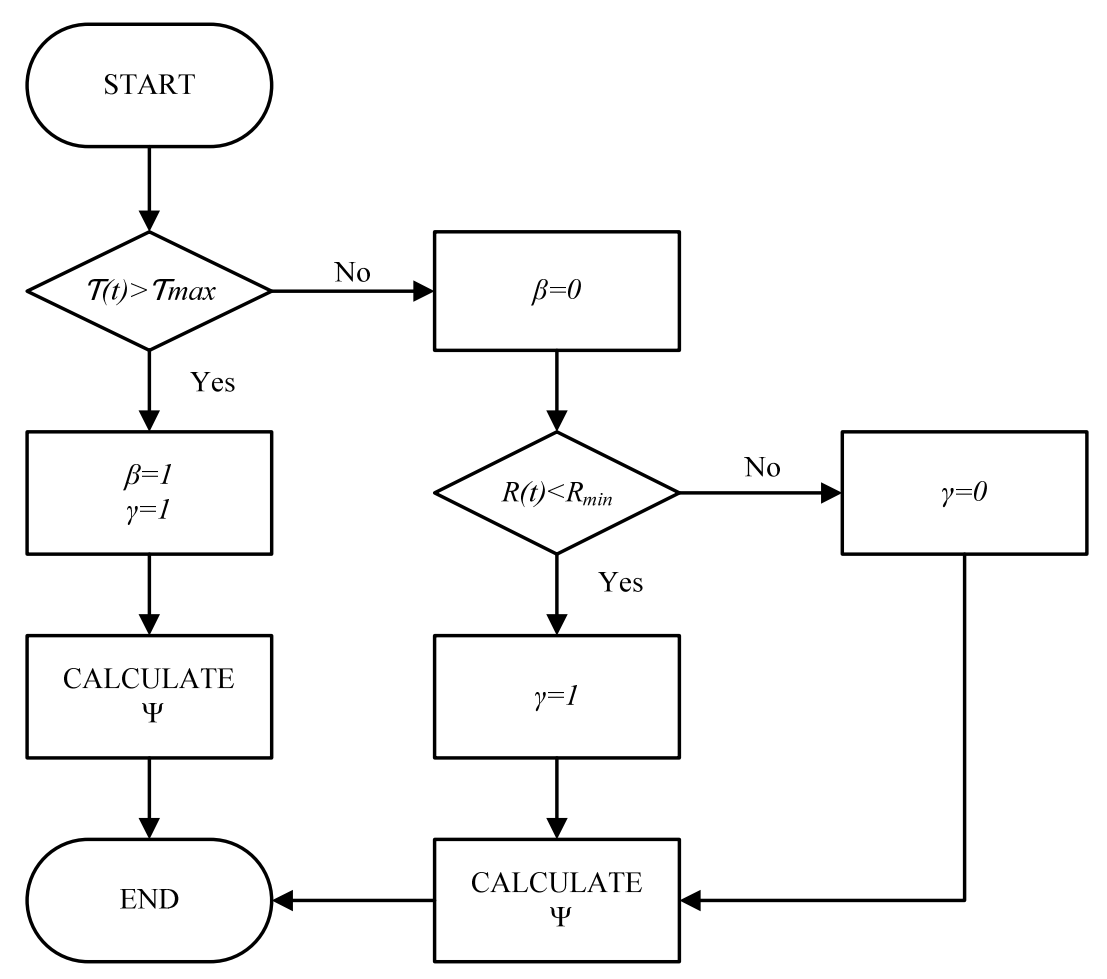

Figure 1. Priority flowchart

Let us consider three situations.

(1) If current slot delay is high, it means, $\tau(t)>\tau_{\max }$, where $\tau(t)$ and $\tau_{\max }$ are the current delay and maximum delay respectively, then according to our consideration we will set $\gamma=0$, and $\beta=1$.

(2) If current slot delay is ok, means $\tau(t)<\tau_{\max }$, but current slot DR is failed to achieve minimum DR, means 
$R(t)<R_{\min }$, then according to our consideration we will set $\gamma=1, \beta=0$.

(3) If current slot delay is ok, means $\tau(t)<\tau_{\max }$ and current slot DR achieve its minimum DR, means $R(t)>R_{\min }$, where $R(t)$ is the current bit rate, then according to our consideration we will set $\gamma=0, \beta=0$. Let us consider the first situation. Suppose, $\tau(t)=2 \tau_{\max }$. Now from the priority equation,

$$
\Psi=\left[\left[\left(\frac{R_{\min }}{R(t)}\right)^{\gamma} *\left\{\frac{\tau(t)}{\tau(t-1) * \tau_{\max }}\right\}^{\beta}\right]+\beta\right]^{\Delta}
$$

Now,

$$
\Psi=\left[\left[\left(\frac{R_{\min }}{R(t)}\right)^{\gamma} *\left\{\frac{2 \tau_{\max }}{\tau(t-1)^{*} \tau_{\max }}\right\}^{\beta}\right]+\beta\right]^{\Delta}
$$

Now putting the values $\gamma=0$ and $\beta=1$ in Equation (12) we get:

$$
\Psi=\left[\left[\left(\frac{R_{\min }}{R(t)}\right)^{0} *\left\{\frac{2 \tau_{\max }}{\tau(t-1) * \tau_{\max }}\right\}^{1}\right]+1\right]^{1}
$$

So we can see that the value of $\Psi$ must be greater than 1, which clearly shows that time delay is given the highest priority.

Now considering the second situation, suppose $R_{\min }=2 R(t)$, therefore we get:

$$
\Psi=\left[\left[\left(\frac{2 R(t)}{R(t)}\right)^{\gamma} *\left\{\frac{\tau(t)}{\tau(t-1) * \tau_{\max }}\right\}^{\beta}\right]+\beta\right]^{\Delta}
$$

Now putting the values $\gamma=1$ and $\beta=0$ in Equation (14), we get:

$$
\Psi=\left[\left[\left(\frac{2 R(t)}{R(t)}\right)^{1} *\left\{\frac{\tau(t)}{\tau(t-1) * \tau_{\max }}\right\}^{0}\right]+0\right]^{1}
$$

The value of $\Psi$ will be greater than 1 . This shows that if minimum bit-rate is not achieved, that user will get priority.

Now considering the third situation, suppose $2 R_{\min }=R(t)$ and $2 \tau(t)=\tau_{\max }$ :

$$
\Psi=\left[\left[\left(\frac{R(t)}{2 R(t)}\right)^{\gamma} *\left\{\frac{2 \tau(t)}{\tau(t-1) * \tau_{\max }}\right\}^{\beta}\right]+\beta\right]^{\Delta}
$$

Now putting the values $\gamma=0$ and $\beta=0$ in Equation (16), we get:

$$
\Psi=\left[\left[\left(\frac{R(t)}{2 R(t)}\right)^{0} *\left\{\frac{2 \tau(t)}{\tau(t-1) * \tau_{\max }}\right\}^{0}\right]+0\right]^{1}
$$

The value of $\Psi$ will be equal to 1 , which means that this user is under ideal conditions and hence requires no additional priority.

\subsection{Power Allocation Technique}

We allocate resources according to the priority. First we determine the priority. And then allocate subset of sub-carriers to the user from sub-carrier set $\phi$. After that we allocate power using the MLWFA. And then we allocate the rest of the sub-carrier set to those users who are unable to meet their minimum data rate using the conventional schemes.

The power allocation of optimization problem of MC users can be stated as: 


$$
\begin{aligned}
\text { Maximize } & \sum_{k} \sum_{n} B_{k, n} \\
\text { subject to } & \sum_{k} \sum_{n} P_{k, n} \leq \sum P_{k} \\
& P_{k, n}>0 ; P_{k, n} f^{2} \leq I_{\max } \\
& B_{k, n}=\log _{2}\left(1+\frac{P_{k, n} G D^{-\alpha_{m}}}{\sigma^{2}+I}\right)
\end{aligned}
$$

Using the Lagrangian function,

$$
L=\log _{2}\left(1+\frac{P_{k, n} G D^{-\alpha_{m}}}{\sigma^{2}+I}\right)+\lambda P_{k}
$$

The derivation of Equation (19) with respect to $P_{k}$ is given by,

$$
\frac{\delta L}{\delta P_{k}}=\frac{\frac{G D^{-\alpha_{m}}}{\sigma^{2}+I}}{\log _{2}\left(1+\frac{P_{k, n} G D^{-\alpha_{m}}}{\sigma^{2}+I}\right)}+\lambda=0
$$

$n \in$ sub-carrier set $\phi$.

From Equation (20) using Karush-Kuhn-Tucker conditions and setting the equation zero we can obtain,

$$
\frac{A_{k, n}}{1+\frac{P_{k, n} G_{n} D^{-\alpha_{n}}}{\sigma^{2}+I}}=\frac{A_{k, n_{1}}}{1+\frac{P_{k, n_{1}} G_{n_{1}} D^{-\alpha_{n_{1}}}}{\sigma^{2}+I}}
$$

Where $A_{k, n}=\frac{P_{k, n} G_{n} D^{-\alpha_{n}}}{\sigma^{2}+I}, A_{k, n_{1}}=\frac{P_{k, n_{1}} G_{n_{1}} D^{-\alpha_{n_{1}}}}{\sigma^{2}+I}$ and $n, n_{1} \in \phi$ and $n \neq n_{1}$.

Equation (21) can be written as,

$$
P_{k, n}=P_{k, n_{1}}+\frac{A_{k, n}-A_{k, n_{1}}}{A_{k, n} * A_{k, n_{1}}}
$$

From Equation (22), taking into consideration $P_{k, n}>0$ and $P_{k, n} \leq P_{k}$ we have,

$$
P_{k, n}=\left[\frac{1}{n_{k}}\left(P_{k}+\sum_{n=0}^{n_{k}} A_{k, n}\right)-A_{k, n}\right]
$$

Now the optimization problem for the FC users can be stated as:

$$
\begin{aligned}
& \text { Maximize } \sum_{k} \sum_{n} b_{k, n} \\
& \text { subject to } \sum_{k} \sum_{n} p_{k, n} \leq \sum P_{k} \\
& p_{k, n} g^{2} \leq I_{\max } \\
& b_{k, n}=\log _{2}\left(1+\frac{p_{k, n} g d^{-\alpha_{F}}}{\sigma^{2}+I_{F}}\right)
\end{aligned}
$$


Using Lagrangian function,

$$
L=\log _{2}\left(1+\frac{p_{k, n} g d^{-\alpha_{F}}}{\sigma^{2}+I_{F}}\right)+\lambda P_{k}
$$

The derivation of Equation (25) with respect to $P_{k}$ is given by,

$$
\frac{\delta L}{\delta p_{k}}=\frac{\frac{g d^{-\alpha_{F}}}{\sigma^{2}+I_{F}}}{\log _{2}\left(1+\frac{p_{k, n} g d^{-\alpha_{F}}}{\sigma^{2}+I_{F}}\right)}+\lambda=0
$$

$n \in$ sub-carrier set $\phi$.

From Equation (26) using Karush-Kuhn-Tucker conditions and setting the equation zero we can obtain, where,

$$
\frac{E_{k, n}}{1+\frac{p_{k, n} g_{n} d^{-\alpha_{F}}}{\sigma^{2}+I_{F}}}=\frac{E_{k, n_{1}}}{1+\frac{p_{k, n_{1}} g_{n_{1}} d^{-\alpha_{n_{1}}}}{\sigma^{2}+I_{F}}}
$$

Equation (27) can also be written as,

$$
p_{k, n}=p_{k, n_{1}}+\frac{E_{k, n}-E_{k, n_{1}}}{E_{k, n} * E_{k, n_{1}}}
$$

And hence from Equation (28), we can therefore state that,

$$
p_{k, n}=\left[\frac{1}{n_{k}}\left(p_{k}+\sum_{n=0}^{n_{k}} E_{k, n}\right)-E_{k, n}\right]
$$

In our model we will allocate power and subcarrier in the following way. Firstly the system will seek out the entire active MUs' within the network coverage area and sort them out in a list. Then by setting the value of $k=1$, we are now considering the first user. Then we calculate the priority factor of this user. If $\Psi$ is greater than 1 then we allocate the best subcarrier set available to that user. If vice-versa then we allocate the normal subcarrier set to that user. This process is repeated for every user by increasing the value of $k$ by 1 every time, while continuously checking whether we have any remaining subcarriers remaining or not. This process loop will only seize to continue if either of the conditions are broken. Afterwards we allocate power using MLWFA. After allocating power we calculate the DR for the users. Then we check again for remaining subcarriers, and if there are any remaining, then we allocate power to those users whose DR is less than $R_{\min }$ using conventional power allocation schemes. But if there are no more remaining sub-carrier sets, then we simply end the process. The entire process is better demonstrated by Figure 2. 


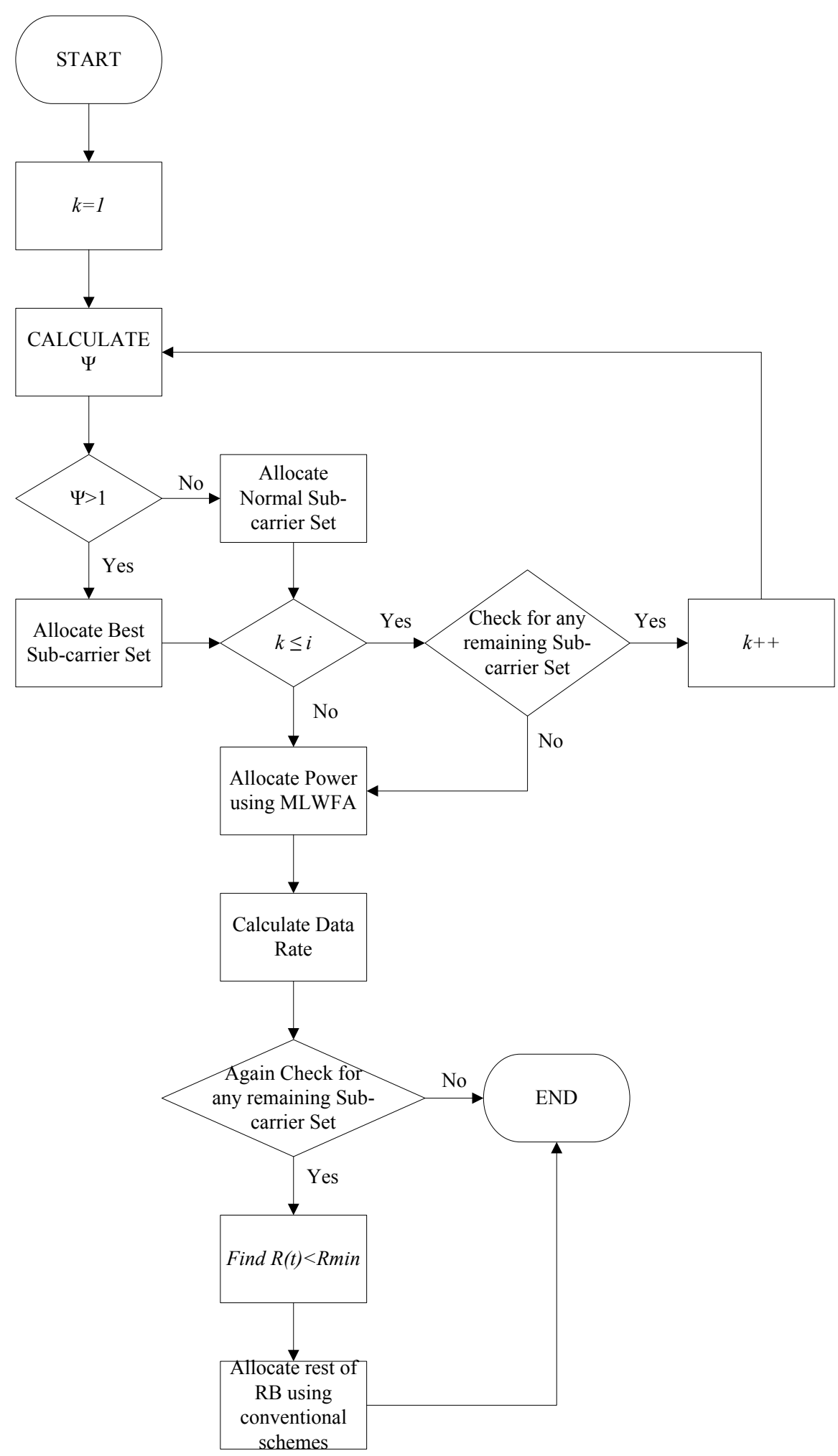

Figure 2. Resource allocation flowchart

\section{Simulation and Results}

In Figure 3, it shows the effect of FC transmit power on outage probability of MC and FCs. Here the circle marked straight line represents the outage probability of MC using optimal power allocation when 50 FCs are deployed in the system. We can see here that the outage probability increases with FC transmit power. This 
occurs due to the additional CCI caused by the FCs. The circle marked dashed line which represents the outage probability of the MC while using our proposed model, also behaves in the same way but its outage has slightly increased since we have introduced fairness into our system. In the figure we can also see that if the number of FCs increases in the system, the outage probability also increases as shown by the diamond marked straight and dashed lines. Now heading over to the reading for the FCs, the square marked straight line shows the outage probability of the FCs while using optimal power allocation and the square marked dashed line represents the outage probability of FCs of the proposed system model. We can clearly see here that in proposed system, the outage probability improves significantly. Similar behavior is shown when the number FCs deployed in the system is doubled, as shown by both the star marked straight and dashed lines. So we can say that the outage probability of the total system is bound to improve.

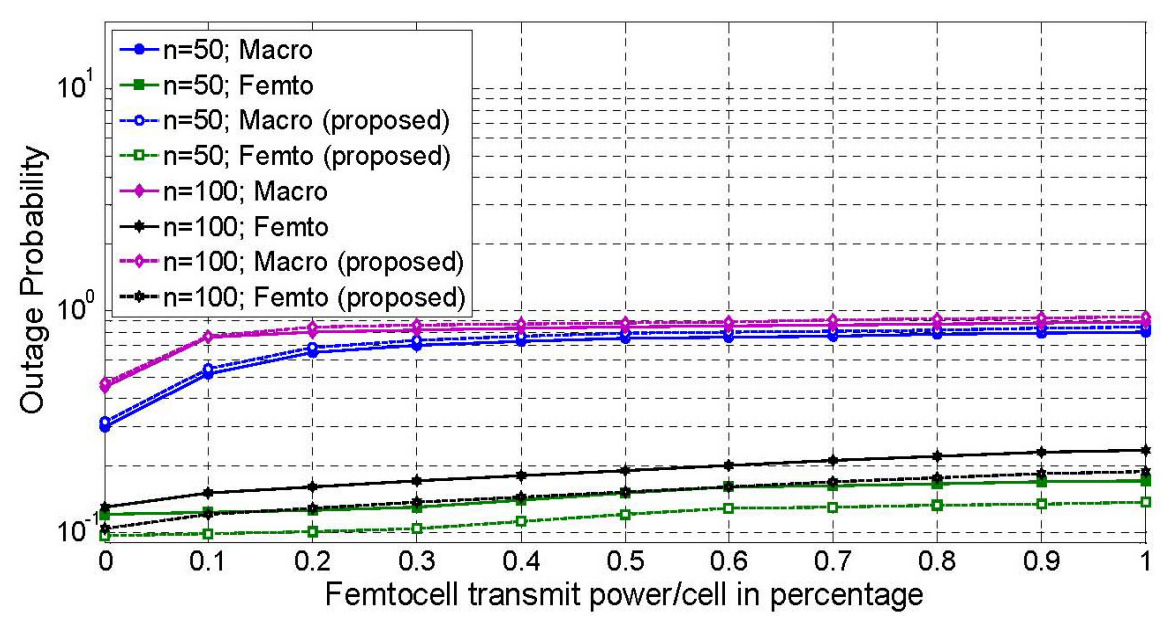

Figure 3. Effects of Femto-Cell transmit power on the system's outage probability

Figure 4 shows the effect of FC transmit power on FC throughput per cell. Here we can see that by increasing the transmission power of FC, the throughput of all the FCs in the system increases. In the figure, the circle marked straight line denotes the throughput of the optimal power allocation and the circle marked dashed line denotes the proposed model throughput. We can see that the proposed model shows a slight improvement. The figure also shows that when we increase the number of FCs in the system, it maintains a similar behavior as shown by both the square marked straight and dashed lines, but the FCs throughput decreases. This occurs as the interference increase with the increase in the number of FCs as per Equation (3).

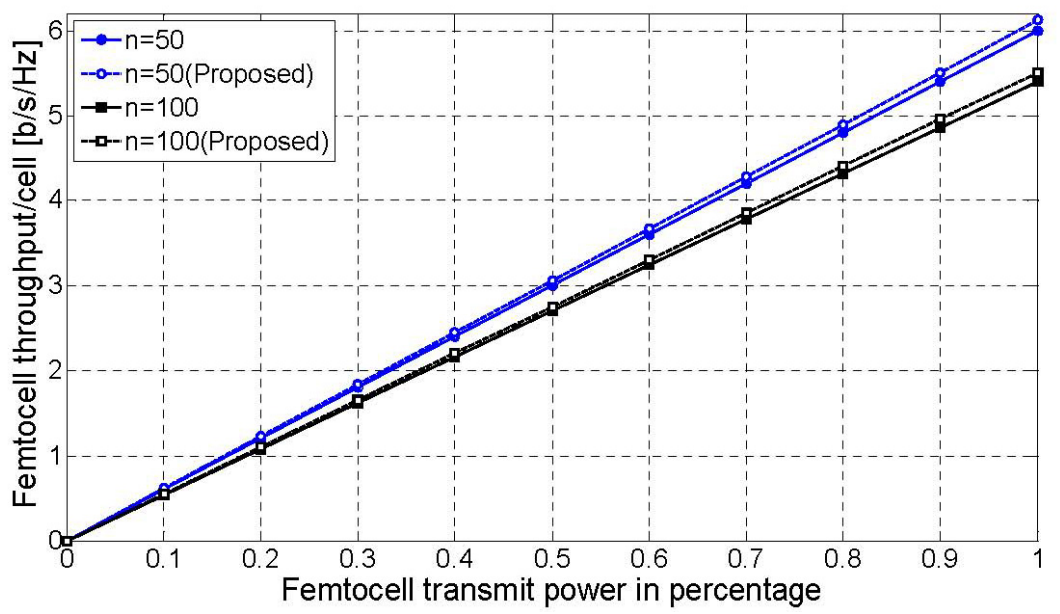

Figure 4. Effects of Femto-Cell transmit power on Femto-Cell Throughput Per Cell 
Figure 5 represents the FC transmit power on MC throughput per cell. In contrast to the previous figure, here the throughput of the MC in the system decreases. This is due to the existence of FCs in the system. From equation (1), we see that increase in the power of the FC increases interference. Due to that the MC system throughput decreases. The circle marked straight line is the throughput while using the optimal power allocation and the circle marked dashed line represents our proposed power allocation model. The reason why our model gives lesser throughput is because the optimal power allocation concept is based on giving the best subcarrier to the best users. But since we are maintaining fairness amongst users (e.g., cell-edge users and users near the BS), this results in a slight decrease in the MC throughput of our proposed system. Similar behavior is seen when the number of FCs in the system increases. We can see in the figure above that all the curves behaves in a way so that they achieve a minimum data rate, which has been ensured due to the introduction of our proposed priority parameter.

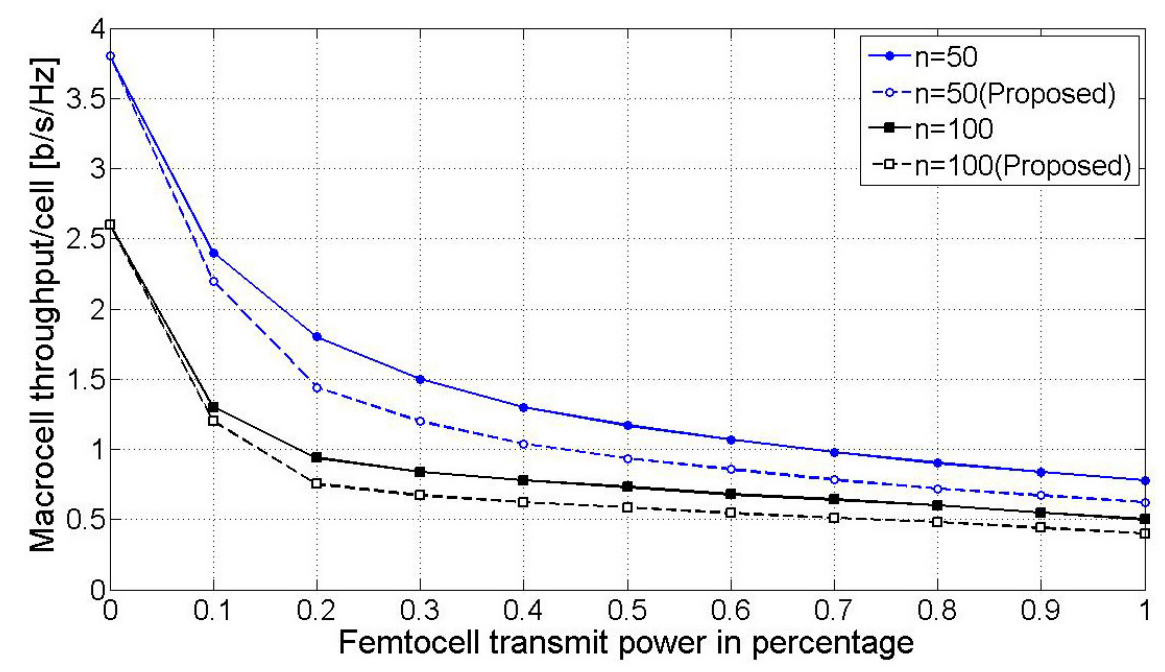

Figure 5. Effects of Femto-Cell transmit power on Macro-Cell throughput per cell

Figure 6 represents the effect of FC transmit power on the total throughput per cell. From this figure we can see that the total system throughput ultimately increases as the power of the FCs are increases as well. Here the circle marked straight line represents the output while using the optimal power allocation and the circle marked dashed line represents the output of our proposed model. The square marked straight lines and square marked dashed lines represents the results if the total number of FCs were increased to 100 . These results clearly indicate that the total system throughput remains similar in comparison to that of the optimal power allocation.

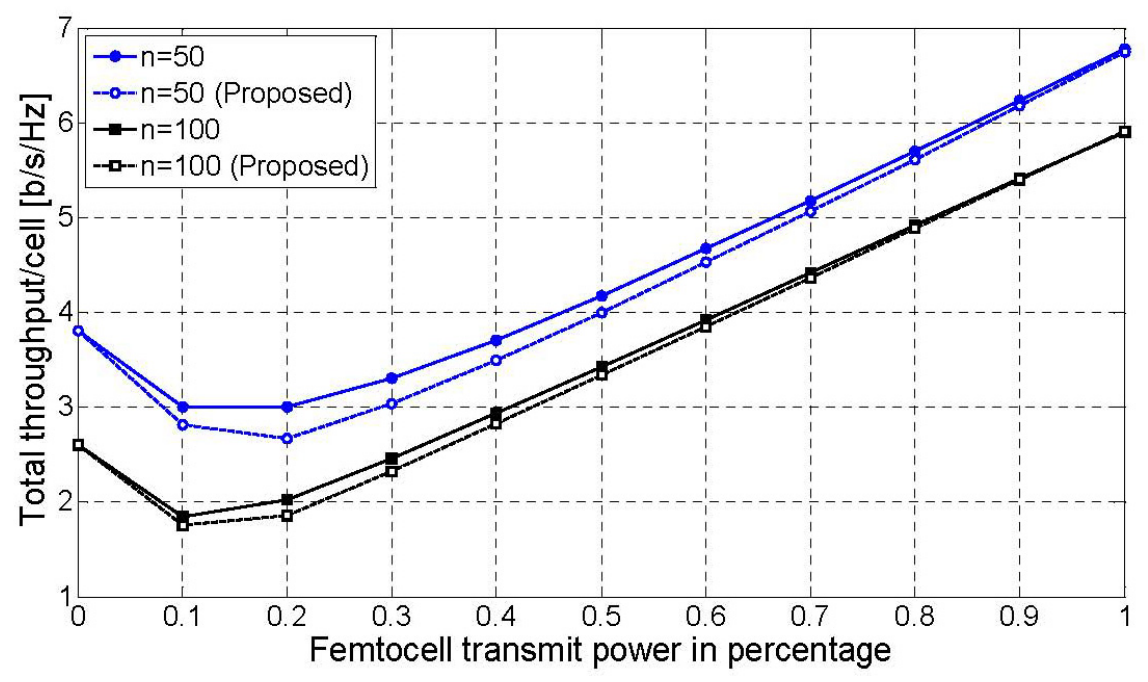

Figure 6. Effects of Femto-Cell transmit power on total throughput per cell 


\section{Conclusion}

In this work, we used a suboptimal power allocation algorithm using priority parameter. The primary motivation for our work is to maintain fairness amongst all the users while not compromising the total system throughput. The results of our work have been compared with the results of the optimal power allocation model used in different researches. Our proposed model proved to be better because we successfully achieved improved outage probability while maintaining the total system throughput. We also managed to do so while maintaining fairness amongst all the users in the system by introducing a priority parameter. The channel model considered in this work is Rayleigh fading model which is not exactly applicable for the $3 \mathrm{G}$ and $4 \mathrm{G}$ cellular systems. We would like to extend this work by considering Nakagami-m fading channel for the optimal subcarrier-power pair.

\section{References}

Ahamed, V. T., Karthick, N. G., \& Joseph, P. K. (2008). Effect of mobile phone radiation on heart rate variability. Comput. Biol. Med, 38, 709-712. http://dx.doi.org/10.1016/j

Ahmed, M. H., Anpalagan, A., Chen, K. C., Han, Z., \& Hossain, E. (2009). Fairness in Radio Resource Management for Wireless Networks. EURASIP Journal on Wireless Communications and Networking, 2009, 1-3. http://dx.doi.org/10.1155/2009/642878

Assaad, M., \& Mourad, A. (2008). New Frequency-Time Scheduling Algorithms for 3GPP/LTE-like OFDMA Air Interface in the Downlink, IEEE VTC Spring 2008, pp. 1964-1969. http://dx.doi.org/10.1109/VETECS.2008.444

Boudec, J. L. (2009). Rate Adaptation, Congestion Control and Fairness: A Tutorial. EPFL Lausanne. http://dx.doi.org/10.1234/12345678

Elayoubi, E., Haddada, O. B., \& Fourestie, B. (2008). Performance Evaluation of Frequency Planning Schemes in OFDMA based Networks. IEEE Transactions on Wireless Communications, 7, 1623-1633. http://dx.doi.org/10.1109/TWC.2008.060458

Flanagan, M., \& Fagan, A. D. (2003). A Simple Proof of the Water Filling Theorem for Multichannel Modulation. Department of Electronic and Electrical Engineering University College Dublin, Bel eld, Dublin.

Holger, C. (2008). Co-Channel Operation of Macro- and Femtocells in a Hierarchical Cell Structure. Int $J$ Wireless Inf Networks, 15, 137-147. http://dx.doi.org/10.1109/PIMRC.2007.4394515

IEEE 802.16m-07/002r4, TGm System Requirements Document (SRD), approved 19 Oct 20072011.

Juang, R. T., Ting, P., Lin, H. P., \& Lin, D. B. (2010). Interference Management of Femtocell in Macrocellular Networks. Wireless Telecommunications Symposium (WTS), $2010, \quad 1-4$. http://dx.doi.org/10.1109/WTS.2010.5479646

Kaiser, M. S., \& Ahmed, K. M. (2010). Radio Resource Allocation for Heterogeneous Services in Relay Enhanced OFDMA Systems. Journal of Communications, 5, 447-454. http://dx.doi.org/10.4304/jcm.5.6.445-446

Max-Min Fairness. (2011) In Wikipedia, the free encyclopedia. Retrieved from http://en.wikipedia.org/wiki/2011

Necker, M. C. (2007). Local Interference Coordination in Cellular OFDMA Networks, IEEE VTC (F) 07 pp. 1741-1746. http://dx.doi.org/10.1109/VETECF.2007.368

Rengarajan, B., Stolyar, A., \& Viswanathan, H. (2010). Self-Organizing Dynamic Fractional Frequency Reuse in OFDMA Systems. IEEE Conference on Computer Communications, 2010, 691-699. http://dx.doi.org/10.1109/INFOCOM.2008.119

Revermann, C. (2002). Health and ecological aspects of mobile telecommunications and transmitters. Retrieved from http://www.tab-beimbundestag.de/en/publications/reports/ab082.html

Simonsson, A. (2007). Frequency Reuse and Intercell Interference Co-Ordination in E-UTRA. IEEE VTC(S), 2007, 3091- 3095. http://dx.doi.org/10.1109/VETECS.2007.633

Yeh, S., Talwar, S., Lee, S., \& Kim, H. (2008). WiMAX Femtocells: A Perspective on Network Architecture, Capacity, and Coverage. IEEE Communications Magazine, 46, 58-65. http://dx.doi.org/10.1109/MCOM.2008.4644120

Yin, H., \& Alamouti, S. (2006). OFDMA: A Broadband Wireless Access Technology. IEEE Proc. of Sarnok Symposium, 2006, 1-4. http://dx.doi.org/10.1109/SARNOF.2006.4534773 
Yu, W., \& Cion, J. M. (2001). On Constant Power Water-filling. IEEE ICC, 2001, 1665-1669. http://dx.doi.org/10.1109/ICC.2001.937077

Zhou, Z., \& Sato, T. (2009) A Single Antenna Interference Cancellation Algorithm for OFDM Communication Systems, Graduate School of Global Information and Telecommunication Studies Waseda University. http://dx.doi.org/10.1109/VETECS.2009.5073344

Zou, H., Chowdhery, A., \& Cion, J. M. (2010). A Centralized Multi-Level Water-Filling Algorithm for Dynamic Spectrum Management, Stanford University Department of Electrical Engineering Stanford, CA 94305 USA 2010. http://dx.doi.org/10.1109/ACSSC.2009.5470058 